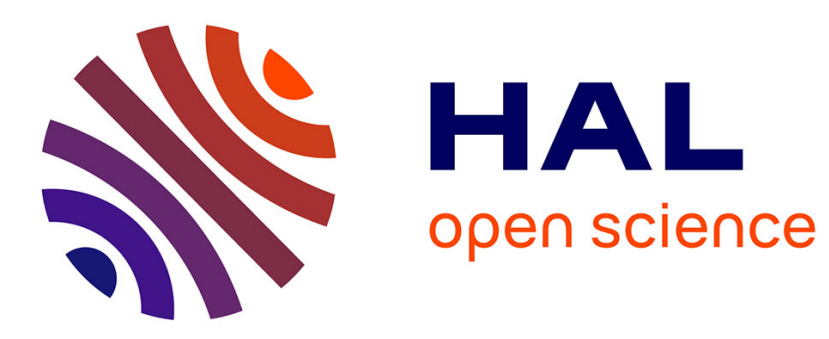

\title{
Finite volume method for nonlinear transmission problems
}

Franck Boyer, Florence Hubert

\section{To cite this version:}

Franck Boyer, Florence Hubert. Finite volume method for nonlinear transmission problems. Proceedings of 17th International Conference on Domain Decomposition Methods, Jul 2006, Strobl, Austria. hal-00178005

\section{HAL Id: hal-00178005 https://hal.science/hal-00178005}

Submitted on 9 Oct 2007

HAL is a multi-disciplinary open access archive for the deposit and dissemination of scientific research documents, whether they are published or not. The documents may come from teaching and research institutions in France or abroad, or from public or private research centers.
L'archive ouverte pluridisciplinaire HAL, est destinée au dépôt et à la diffusion de documents scientifiques de niveau recherche, publiés ou non, émanant des établissements d'enseignement et de recherche français ou étrangers, des laboratoires publics ou privés. 


\title{
Finite volume method for nonlinear transmission problems
}

\author{
Franck BOYER ${ }^{1}$ and Florence HUBERT ${ }^{2}$ \\ 1 39, rue F. Joliot Curie, 13453 Marseille Cedex 13, FRANCE \\ fboyer@cmi.univ-mrs.fr \\ 2 fhubert@cmi.univ-mrs.fr
}

\section{Introduction}

Discrete Duality Finite Volume (DDFV) schemes have recently been developped to approximate monotone nonlinear diffusion problems

$$
-\operatorname{div}(\varphi(z, \nabla u(z)))=f(z), \text { in } \Omega, u=0, \text { on } \partial \Omega,
$$

on general 2D grids. The principle of such schemes is to introduce discrete unknowns both at centers and vertices of any given primal mesh. A discrete gradient operator is then built over the diamond cells associated to the mesh and finally, the discrete flux balance equations are written on the primal and dual control volumes (see Section 2). The main advantages of this approach is that few geometric assumptions are needed for the grid (non conformal grids are allowed for instance), and that the discrete problem inherits the main properties (monotonicity, symmetry, ...) of the continuous one. In Andreianov et al. [2007], it is proved that the scheme is well-posed and convergent. Under suitable regularity assumptions on $\varphi$ and $u$, some error estimates are also obtained.

Application of these schemes to nonlinear transmission problems, that is when $\varphi$ presents some discontinuities with respect to the space variable $z$, were first investigated in Boyer and Hubert in the case where uniform growth and coercivity conditions for $\xi \mapsto \varphi(z, \xi)$ are assumed to hold over the domain.

We propose here to generalize this analysis to the case where these growth and coercivity conditions are no more uniform on the domain. We can imagine for instance that $\varphi$ is linear with respect to $\xi$ on a subdomain and fully nonlinear on its complementary. Such situations arise for instance in bimaterial problems in elastic-plastic mechanics (see Feistauer and Sobotíková [1990], Liu [1999], Liu [2000]).

Let us precise the situation under study. Let $\Omega$ be a bounded polygonal open set in $\mathbb{R}^{2}$, split into $N$ open polygonal subdomains $\Omega_{i}$ : 


$$
\bar{\Omega}=\cup_{i=1}^{N} \overline{\Omega_{i}}, \Omega_{i} \cap \Omega_{j}=\emptyset \text { if } i \neq j,
$$

and that $\varphi: \Omega \times \mathbb{R}^{2} \rightarrow \mathbb{R}^{2}$ in equation (1) is a Caratheodory function, constant with respect to $z$ on each $\Omega_{i}: \varphi(z, \xi)=\varphi_{i}(\xi)$, for all $z \in \Omega_{i}$ and $\xi \in \mathbb{R}^{2}$. There exists a familly $\left.\boldsymbol{p}=\left(p_{i}\right)_{\{i=1, \cdots, N\}}, p_{i} \in\right] 1, \infty\left[\right.$ and a constant $C_{\varphi}>0$ such that

- Monotonicity on each subdomain $\Omega_{i}$ : for all $(\xi, \eta) \in \mathbb{R}^{2} \times \mathbb{R}^{2}$

$$
\begin{aligned}
& \left(\varphi_{i}(\xi)-\varphi_{i}(\eta), \xi-\eta\right) \geq C_{\varphi}|\xi-\eta|^{2}\left(1+|\xi|^{p_{i}}+|\eta|^{p_{i}}\right)^{\frac{p_{i}-2}{p_{i}}}, \text { if } p_{i} \leq 2 . \\
& \left(\varphi_{i}(\xi)-\varphi_{i}(\eta), \xi-\eta\right) \geq C_{\varphi}|\xi-\eta|^{p_{i}}, \text { if } p_{i}>2 .
\end{aligned}
$$

- Coercitivity on each subdomain $\Omega_{i}$ : for all $\xi \in \mathbb{R}^{2}$

$$
\left(\varphi_{i}(\xi), \xi\right) \geq C_{\varphi}\left(|\xi|^{p_{i}}-1\right) .
$$

- Growth conditions : for all $(\xi, \eta) \in \mathbb{R}^{2} \times \mathbb{R}^{2}$,

$$
\begin{aligned}
& \left|\varphi_{i}(\xi)-\varphi_{i}(\eta)\right| \leq C_{\varphi}|\xi-\eta|^{p_{i}-1}, \text { if } p_{i} \leq 2, \\
& \left|\varphi_{i}(\xi)-\varphi_{i}(\eta)\right| \leq C_{\varphi}\left(1+|\xi|^{p_{i}-2}+|\eta|^{p_{i}-2}\right)|\xi-\eta|, \text { if } p_{i}>2 .
\end{aligned}
$$

Remark that assumption $\left(\mathcal{H}_{3}\right)$ implies that

$$
\left|\varphi_{i}(\xi)\right| \leq C_{\varphi}\left(|\xi|^{p_{i}-1}+1\right), \quad \forall \xi \in \mathbb{R}^{2} .
$$

We introduce $L^{\boldsymbol{p}}(\Omega)=\left\{u / u_{\left.\right|_{\Omega_{i}}} \in L^{p_{i}}\left(\Omega_{i}\right)\right\}, W_{0}^{1, \boldsymbol{p}}(\Omega)=\left\{u \in W_{0}^{1,1}(\Omega) / \nabla u \in\right.$ $\left.\left(L^{\boldsymbol{p}}(\Omega)\right)^{2}\right\}$, and for $\boldsymbol{q}=\left(q_{i}\right)_{i=1, \cdots, N}$, we denote $\|u\|_{L^{p}}^{\boldsymbol{q}}=\sum_{i=1}^{N}\left\|u_{\left.\right|_{\Omega_{i}}}\right\|_{L^{p_{i}\left(\Omega_{i}\right)}}^{q_{i}}$. We finally note $\boldsymbol{p}_{\min }=\min \left(p_{i}\right)$ and $\boldsymbol{p}_{\max }=\max \left(p_{i}\right)$.

Theorem 1 (Liu [1999]). Under assumptions $\left(\mathcal{H}_{1}\right),\left(\mathcal{H}_{2}\right),\left(\mathcal{H}_{4}\right)$, the problem (1) admits for all $f \in L^{\boldsymbol{p}_{\min }^{\prime}}(\Omega)$ a unique solution $u \in W_{0}^{1, \boldsymbol{p}}(\Omega)$.

These problems can be approximated either by finite element method, whose study is undertaken in particular in Liu [2000], or by the m-DDFV ("modified" Discrete Duality Finite Volume) method developped for nonlinear elliptic equations with discontinuities in Boyer and Hubert.

\section{The m-DDFV scheme}

Let $\mathfrak{M}_{i}$ be a finite volume mesh on $\Omega_{i}$ for $i=1, \cdots, N$ and $\mathfrak{M}=\cup_{i=1}^{N} \mathfrak{M}_{i}$. Note that the mesh $\mathfrak{M}$ can present non standard edges in particular on the boundaries $\partial \Omega_{i} \cap \partial \Omega_{j}$. We associate to each control volume $\mathcal{K} \in \mathfrak{M}$ a point $x_{\mathcal{K}} \in \mathcal{K}$, called the center. Let $\mathfrak{M}^{*}$ be the dual mesh of $\mathfrak{M}$, that is the mesh whose control volumes $\mathcal{K}^{*} \in \mathfrak{M}^{*}$ are obtained by joining the centers of control 
volumes around a vertice $x_{\mathcal{K}^{*}}$ (see Fig. 1$)$. Note $\mathcal{T}=\left(\mathfrak{M}, \mathfrak{M}^{*}\right)$. The DDFV methods involve both unknowns $\left(u_{\mathcal{K}}\right) \in \mathbb{R}^{\mathfrak{M}}$ on $\mathfrak{M}$ and $\left(u_{\mathcal{K}^{*}}\right) \in \mathbb{R}^{\mathfrak{M}^{*}}$ on $\mathfrak{M}^{*}$, we note $u^{\mathcal{T}}=\left(u_{\mathcal{K}}, u_{\mathcal{K}^{*}}\right) \in \mathbb{R}^{\mathfrak{M}} \times \mathbb{R}^{\mathfrak{M}^{*}}$. Integrating equation (1) on both $\mathcal{K} \in \mathfrak{M}$ and $\mathcal{K}^{*} \in \mathfrak{M}^{*}$, the classical DDFV scheme consists in approaching the nonlinear fluxes $\int_{\partial \mathcal{K}}\left(\boldsymbol{\varphi}(z, \nabla u(z)), \boldsymbol{\nu}_{\mathcal{K}}\right) d z$ and $\int_{\partial \mathcal{K}^{*}}\left(\varphi(z, \nabla u(z)), \boldsymbol{\nu}_{\mathcal{K}^{*}}\right) d z$ by using a discrete gradient $\nabla^{\mathcal{T}} u^{\mathcal{T}}$, piecewise constant on a partition $\mathfrak{D}=$ $(\mathcal{D})_{\mathcal{D} \in \mathcal{D}}$ called the diamond cells, and $\varphi_{\mathcal{D}}\left(\nabla_{\mathcal{D}}^{\mathcal{T}} u^{\mathcal{T}}\right)=\frac{1}{|\mathcal{D}|} \int_{\mathcal{D}} \varphi\left(z, \nabla_{\mathcal{D}}^{\mathcal{T}} u^{\mathcal{T}}\right) d z$. Each diamond cell is a quadrangle whose diagonals are some edge $\sigma=\mathcal{K} \mid \mathcal{L}$ and the edge $\sigma^{*}=\left(x_{\mathcal{K}}, x_{\mathcal{L}}\right)$. The set $\mathfrak{D}_{\Gamma_{i j}}$ specifies the diamond cells lying
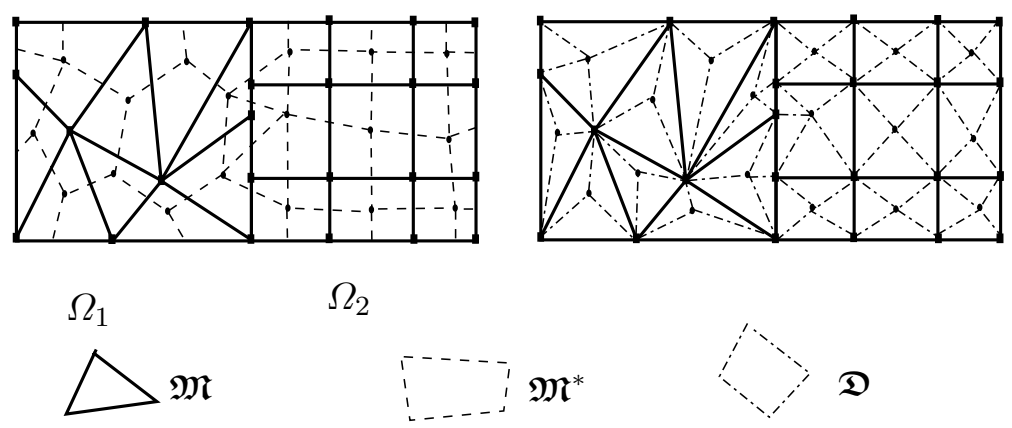

$\mathfrak{D}$

Fig. 1. The three meshes $\mathfrak{M}, \mathfrak{M}^{*}, \mathfrak{D}$

across two distinct subdomains $\Omega_{i}$ and $\Omega_{j}$ and $\mathfrak{D}_{\Gamma}=\cup_{\substack{i, j \\ i \neq j}} \mathfrak{D}_{\Gamma_{i j}}$.

The discrete gradient introduced in Coudière et al. [1999], Hermeline [2003], Domelevo and Omnes [2005] reads

$$
\nabla^{\mathcal{T}} u^{\mathcal{T}}=\sum_{\mathcal{D} \in \mathfrak{D}} \nabla_{\mathcal{D}}^{\mathcal{T}} u^{\mathcal{T}} 1_{\mathcal{D}}, \nabla_{\mathcal{D}}^{\mathcal{T}} u^{\mathcal{T}}=\frac{1}{\sin \alpha_{\mathcal{D}}}\left(\frac{u_{\mathcal{L}}-u_{\mathcal{K}}}{\left|\sigma^{*}\right|} \boldsymbol{\nu}+\frac{u_{\mathcal{L}^{*}}-u_{\mathcal{K}^{*}}}{|\sigma|} \boldsymbol{\nu}^{*}\right)
$$

with the notations of Fig. 2. The DDFV scheme is then defined by

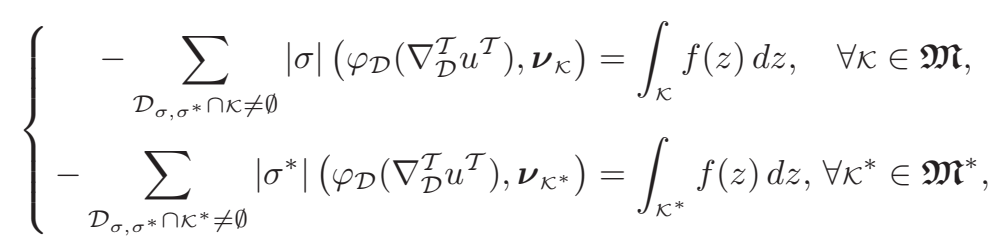

and admits a unique solution. Convergence and error estimates in that case are given in Andreianov et al. [2007] . These error estimates are no more valid as soon as $\varphi_{i} \neq \varphi_{j}$, since we loose the consistency of the nonlinear fluxes across the edges on $\partial \Omega_{i} \cap \partial \Omega_{j}$. To tackle this problem, we proposed in Boyer and Hubert in the case $p_{i}=p_{j}, \forall i, \forall j$ to change the approximation of the nonlinearity on the diamond cells $\varphi_{\mathcal{D}}\left(\nabla_{\mathcal{D}}^{\mathcal{T}} u^{\mathcal{T}}\right)$ into $\varphi_{\mathcal{D}}^{\mathcal{N}}\left(\nabla_{\mathcal{D}}^{\mathcal{T}} u^{\mathcal{T}}\right)$ in such a way 


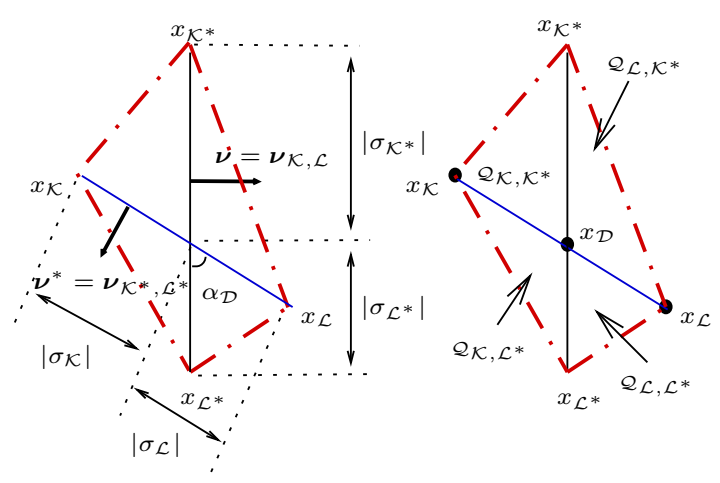

Fig. 2. Notations in a diamond cell $\mathcal{D}=\cup_{\mathcal{Q} \in \mathfrak{Q}_{\mathcal{D}} \mathcal{Q}}$

that enforce the consistency of the fluxes across all the edges. The new scheme reads

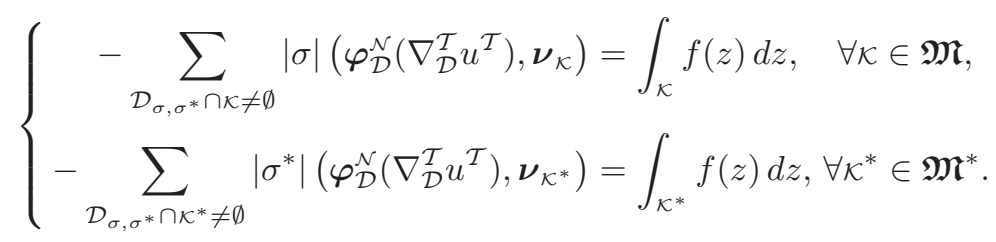

To define $\varphi_{\mathcal{D}}^{\mathcal{N}}\left(\nabla^{\mathcal{T}} u^{\mathcal{T}}\right)$, we introduce a new discrete gradient constant on the quarters $(\mathcal{Q})_{\mathfrak{Q}}$ of the diamond cells (see Fig. 2)

$$
\nabla^{\mathcal{N}} u^{\mathcal{T}}=\sum_{\mathcal{Q} \in \mathfrak{Q}} \nabla_{\mathcal{Q}}^{\mathcal{N}} u^{\mathcal{T}}, \nabla_{\mathcal{D}}^{\mathcal{N}} u^{\mathcal{T}}=\sum_{\mathcal{Q} \in \mathfrak{Q}_{\mathcal{D}}} 1_{\mathcal{Q}} \nabla_{\mathcal{Q}}^{\mathcal{N}} u^{\mathcal{T}}
$$

with $\nabla_{\mathcal{Q}}^{\mathcal{N}} u^{\mathcal{T}}=\nabla_{\mathcal{D}}^{\mathcal{T}} u^{\mathcal{T}}+B_{\mathcal{Q}} \delta_{\mathcal{D}}$, where $\delta_{\mathcal{D}} \in \mathbb{R}^{n_{\mathcal{D}}}$ are artificial unknowns $\left(n_{\mathcal{D}}=\right.$ 4 for interior diamond cells and $n_{\mathcal{D}}=1$ for boundary diamond cells) and $\left(B_{\mathcal{Q}}\right)_{\mathcal{Q} \in \mathfrak{Q}}$ a set of $2 \times n_{\mathcal{D}}$ matrices defined for interior diamond cells by

$$
\begin{aligned}
B_{\mathcal{Q}_{\mathcal{K}, \mathcal{K}^{*}}} & =\frac{1}{\left|\mathcal{Q}_{\mathcal{K}, \mathcal{K}^{*}}\right|}\left(\left|\sigma_{\mathcal{K}}\right| \boldsymbol{\nu}^{*}, 0,\left|\sigma_{\mathcal{K}^{*}}\right| \boldsymbol{\nu}, 0\right), B_{\mathcal{Q}_{\mathcal{L}, \mathcal{L}^{*}}}=\frac{1}{\left|\mathcal{Q}_{\mathcal{L}, \mathcal{L}^{*}}\right|}\left(0,-\left|\sigma_{\mathcal{L}}\right| \boldsymbol{\nu}^{*}, 0,-\left|\sigma_{\mathcal{L}^{*}}\right| \boldsymbol{\nu}\right), \\
B_{\mathcal{Q}_{\mathcal{K}, \mathcal{L}^{*}}} & =\frac{1}{\left|\mathcal{Q}_{\mathcal{K}, \mathcal{L}^{*}}\right|}\left(-\left|\sigma_{\mathcal{K}}\right| \boldsymbol{\nu}^{*}, 0,0,\left|\sigma_{\mathcal{L}^{*}}\right| \boldsymbol{\nu}\right), B_{\mathcal{Q}_{\mathcal{L}, \mathcal{K}^{*}}}=\frac{1}{\left|\mathcal{Q}_{\mathcal{L}, \mathcal{K}^{*}}\right|}\left(0,\left|\sigma_{\mathcal{L}}\right| \boldsymbol{\nu}^{*},-\left|\sigma_{\mathcal{K}^{*}}\right| \boldsymbol{\nu}, 0\right) .
\end{aligned}
$$

Note that $B_{\mathcal{Q}}$ depends only on the geometry of the diamond cell under study.

For $\mathcal{Q} \subset \Omega_{i}$, we note $\varphi_{\mathcal{Q}}(\xi)=\varphi_{i}(\xi)$ and

$$
\boldsymbol{\varphi}_{\mathcal{D}}^{\mathcal{N}}\left(\nabla_{\mathcal{D}}^{\mathcal{T}} u^{\mathcal{T}}\right)=\frac{1}{|\mathcal{D}|} \sum_{\mathcal{Q} \in \mathfrak{Q}_{\mathcal{D}}}|\mathcal{Q}| \varphi_{\mathcal{Q}}\left(\nabla_{\mathcal{Q}}^{\mathcal{N}} u^{\mathcal{T}}\right)
$$

For each $\mathcal{D} \in \mathfrak{D}$, we choose $\delta_{\mathcal{D}} \in \mathbb{R}^{n_{\mathcal{D}}}$ such that, the conservativity of the fluxes is achieved that is 


$$
\begin{aligned}
& \left(\varphi_{\mathcal{Q}_{\mathcal{K}, \mathcal{K}^{*}}}\left(\nabla_{\mathcal{D}}^{\mathcal{T}} u^{\mathcal{T}}+B_{\mathcal{Q}_{\mathcal{K}, \mathcal{K}^{*}}} \delta_{\mathcal{D}}\right), \nu^{*}\right)=\left(\boldsymbol{\varphi}_{\mathcal{Q}_{\mathcal{K}, \mathcal{L}^{*}}}\left(\nabla_{\mathcal{D}}^{\mathcal{T}} u^{\mathcal{T}}+B_{\mathcal{Q}_{\mathcal{K}, \mathcal{L}^{*}}} \delta_{\mathcal{D}}\right), \nu^{*}\right) \\
& \left(\boldsymbol{\varphi}_{\mathcal{Q}_{\mathcal{L}, \mathcal{K}^{*}}}\left(\nabla_{\mathcal{D}}^{\mathcal{T}} u^{\mathcal{T}}+B_{\mathcal{Q}_{\mathcal{L}, \mathcal{K}^{*}}} \delta_{\mathcal{D}}\right), \nu^{*}\right)=\left(\boldsymbol{\varphi}_{\mathcal{Q}_{\mathcal{L}, \mathcal{L}^{*}}}\left(\nabla_{\mathcal{D}}^{\mathcal{T}} u^{\mathcal{T}}+B_{\mathcal{Q}_{\mathcal{L}, \mathcal{L}^{*}}} \delta_{\mathcal{D}}\right), \boldsymbol{\nu}^{*}\right) \\
& \left(\varphi_{\mathcal{Q}_{\mathcal{K}, \mathcal{K}^{*}}}\left(\nabla_{\mathcal{D}}^{\mathcal{T}} u^{\mathcal{T}}+B_{\mathcal{Q}_{\mathcal{K}, \mathcal{K}^{*}}} \delta_{\mathcal{D}}\right), \nu\right)=\left(\varphi_{\mathcal{Q}_{\mathcal{L}, \mathcal{K}^{*}}}\left(\nabla_{\mathcal{D}}^{\mathcal{T}} u^{\mathcal{T}}+B_{\mathcal{Q}_{\mathcal{L}, \mathcal{K}^{*}}} \delta_{\mathcal{D}}\right), \nu\right) \\
& \left(\varphi_{\mathcal{Q}_{\mathcal{K}, \mathcal{L}^{*}}}\left(\nabla_{\mathcal{D}}^{\mathcal{T}} u^{\mathcal{T}}+B_{\mathcal{Q}_{\mathcal{K}, \mathcal{L}^{*}}} \delta_{\mathcal{D}}\right), \boldsymbol{\nu}\right)=\left(\varphi_{\mathcal{Q}_{\mathcal{L}, \mathcal{L}^{*}}}\left(\nabla_{\mathcal{D}}^{\mathcal{T}} u^{\mathcal{T}}+B_{\mathcal{Q}_{\mathcal{L}, \mathcal{L}^{*}}} \delta_{\mathcal{D}}\right), \boldsymbol{\nu}\right)
\end{aligned}
$$

We then only have to solve for each diamond cell in $\mathfrak{D}_{\Gamma}$ a nonlinear problem and $\nabla_{\mathcal{D}}^{\mathcal{N}} u^{\mathcal{T}}$ can be seen as a nonlinear implicit function of $\nabla_{\mathcal{D}}^{\mathcal{T}} u^{\mathcal{T}}$. Note that $\delta_{\mathcal{D}}=0$ as soon as $\mathcal{D} \subset \Omega_{i}$ for some $i=1, \cdots, N$.

Theorem 2. Under assumptions $\left(\mathcal{H}_{1}\right)-\left(\mathcal{H}_{3}\right)$, for all $u^{\mathcal{T}} \in \mathbb{R}^{\mathcal{T}}$ and all diamond cell $\mathcal{D}$, there exists a unique $\delta_{\mathcal{D}}\left(\nabla_{\mathcal{D}}^{\mathcal{T}} u^{\mathcal{T}}\right) \in \mathbb{R}^{n_{\mathcal{D}}}$ satisfying $(6)$. The scheme (4)-(6) admits a unique solution.

For simplicity we state here error estimates obtained when $u$ belongs to the space $E=\left\{u \in \mathcal{C}(\bar{\Omega}), u \in \mathcal{C}^{2}\left(\Omega_{i}\right) \forall i\right\}$, eventhough the result can be extended to the case where $u_{\Omega_{\Omega_{i}}} \in W^{2, p_{i}}\left(\Omega_{i}\right)$. We consider a familly of meshes with convex diamond cells. The geometry's regularity of the meshes is controlled by a quantity denoted by $\operatorname{reg}(\mathcal{T})$, see Boyer and Hubert for more details.

Theorem 3. Assume that the flux $\varphi$ satisfies $\left(\mathcal{H}_{1}\right)-\left(\mathcal{H}_{3}\right)$. Let $f \in L^{\boldsymbol{p}_{\min }^{\prime}}(\Omega)$ and assume that the solution $u$ to (1) belongs to $E$.

There exists $C>0$ depending on $u$, on $\|f\|_{L^{p_{\min }^{\prime}}}$ and on $\operatorname{reg}(\mathcal{T})$ such that

$$
\begin{gathered}
\left\|u-u^{\mathcal{T}}\right\|_{L^{p}}^{\mathbf{2}}+\left\|\nabla u-\nabla^{\mathcal{N}} u^{\mathcal{T}}\right\|_{L^{p}}^{2} \leq C \operatorname{size}(\mathcal{T})^{2\left(\boldsymbol{p}_{\min }-1\right)}, \text { if } \boldsymbol{p}_{\max } \leq 2 \\
\left\|u-u^{\mathcal{T}}\right\|_{L^{p}}^{\boldsymbol{p}}+\left\|\nabla u-\nabla^{\mathcal{N}} u^{\mathcal{T}}\right\|_{L^{p}}^{\boldsymbol{p}} \leq C \operatorname{size}(\mathcal{T})^{\frac{p_{\max }}{\boldsymbol{p}_{\max }-1}}, \text { if } \boldsymbol{p}_{\min } \geq 2
\end{gathered}
$$

As usual, these error estimates (which do not use any geometric assumptions on the solution) are pessimistic and numerical results given in Section 3 show that we can expect a much better behavior of these schemes.

Theorems 2 and 3 can be proved by following similar arguments than the ones presented in Boyer and Hubert for the case $p_{i}=p_{j}, \forall i, \forall j$.

\section{Numerical results}

\subsection{An iterative method to solve the m-DDFV scheme}

We propose to solve the fully nonlinear discrete problem (4)-(6) by the following decomposition-coordination algorithm (see Glowinski [1984], Boyer and Hubert). Let $\mathcal{A}=\left(A_{\mathcal{Q}}\right)_{\mathcal{Q} \in \mathfrak{Q}}$ be a family of definite positive $2 \times 2$ matrices, playing the role of heterogeneous and anisotropic augmented parameters and $\left.\gamma \in] 0, \frac{1+\sqrt{5}}{2}\right]$. The algorithm acts in three steps: 
- Step 1: Find $\left(u^{\mathcal{T}, n}, \delta_{\mathcal{D}}^{n}\right)$ solution of

$$
\begin{aligned}
& \sum_{\mathcal{Q} \in \mathfrak{Q}}|\mathcal{Q}| A_{\mathcal{Q}}\left(\nabla_{\mathcal{D}}^{\mathcal{T}} u^{\mathcal{T}, n}+B_{\mathcal{Q}} \delta_{\mathcal{D}}^{n}-g_{\mathcal{Q}}^{n-1}, \nabla_{\mathcal{D}}^{\mathcal{T}} v^{\mathcal{T}}\right) \\
& =\frac{1}{2} \sum_{\mathcal{K}}|\mathcal{K}| f_{\mathcal{K}} v_{\mathcal{K}}+\frac{1}{2} \sum_{\mathcal{K}^{*}}\left|\mathcal{K}^{*}\right| f_{\mathcal{K}^{*}} v_{\mathcal{K}^{*}}+\sum_{\mathcal{Q} \in \mathfrak{Q}}|\mathcal{Q}|\left(\lambda_{\mathcal{Q}}^{n-1}, \nabla_{\mathcal{D}}^{\mathcal{T}} v\right), \forall v^{\mathcal{T}} \in \mathbb{R}^{\mathcal{T}} \\
& \sum_{\mathcal{Q} \in \mathfrak{Q}_{\mathcal{D}}}|\mathcal{Q}|^{t} B_{\mathcal{Q}} A_{\mathcal{Q}}\left(B_{\mathcal{Q}} \delta_{\mathcal{D}}^{n}+\nabla_{\mathcal{D}}^{\mathcal{T}} u^{\mathcal{T}, n}-g_{\mathcal{Q}}^{n-1}\right)-\sum_{\mathcal{Q} \in \mathfrak{Q}_{\mathcal{D}}}|\mathcal{Q}|^{t} B_{\mathcal{Q}} \lambda_{\mathcal{Q}}^{n-1}=0, \forall \mathcal{D} \in \mathfrak{D}
\end{aligned}
$$

Equation (8) gives, on each $\mathcal{D}$, a formula for $\delta_{\mathcal{D}}^{n}$ as a function of $\nabla_{\mathcal{D}}^{\mathcal{T}} u^{\mathcal{T}, n}$. It follows that (7) is nothing but a global DDFV linear system to solve.

- Step 2: On each $\mathcal{Q}$, find $g_{\mathcal{Q}}^{n} \in \mathbb{R}^{2}$ solution of

$$
\varphi_{\mathcal{Q}}\left(g_{\mathcal{Q}}^{n}\right)+\lambda_{\mathcal{Q}}^{n-1}+A_{\mathcal{Q}}\left(g_{\mathcal{Q}}^{n}-\nabla_{\mathcal{D}}^{\mathcal{T}} u^{\mathcal{T}, n}-B_{\mathcal{Q}} \delta_{\mathcal{D}}^{n}\right)=0 .
$$

This is the unique nonlinear part of the algorithm and can be solved, indepently on each quarter diamond cell, by using the Newton method for instance.

- Step 3: On each $\mathcal{Q}$, compute $\lambda_{\mathcal{Q}}^{n}$ by

$$
\lambda_{\mathcal{Q}}^{n}=\lambda_{\mathcal{Q}}^{n-1}+\gamma A_{\mathcal{Q}}\left(g_{\mathcal{Q}}^{n}-\nabla_{\mathcal{D}}^{\mathcal{T}} u^{\mathcal{T}, n}-B_{\mathcal{Q}} \delta_{\mathcal{D}}^{n}\right)
$$

It is proved in Boyer and Hubert that

Theorem 4. Let $\mathcal{T}$ be a DDFV mesh on $\Omega$. For any family $\left(\boldsymbol{\varphi}_{\mathcal{Q}}\right)_{\mathcal{Q}}$ of strictly monotonic continuous maps from $\mathbb{R}^{2}$ onto itself, for any augmentation matrices family $\mathcal{A}$ and any $\left.\gamma \in] 0, \frac{1+\sqrt{5}}{2}\right]$, the algorithm (7)-(10) converges, when $n$ goes to infinity, towards the unique solution to the $m$-DDFV scheme (4)-(6).

\subsection{Numerical examples}

We illustrate the behaviour of the m-DDFV scheme compared to the DDFV one, on two academic examples for $\Omega=\Omega_{1} \cup \Omega_{2}$ with $\left.\Omega_{1}=\right] 0,0.5[\times] 0,1[$ and $\left.\Omega_{2}=\right] 0.5[\times] 0,1\left[\right.$ and $\varphi_{i}(\xi)=|\xi|^{p_{i}-2} \xi$

$$
\begin{gathered}
\text { Test 1: } u(x, y)=\left\{\begin{array}{l}
x\left(\left(\lambda^{\frac{p_{2}-1}{p_{1}-1}}-1\right)(2 x-1)+1\right) \text { for } x \leq 0.5, \\
(1-x)((1+\lambda)(2 x-1)+1) \text { for } x \geq 0.5,
\end{array}\right. \\
\underline{\text { Test } 2:} u(x, y)=\left\{\begin{array}{l}
\sin (k \pi y)\left(\left(2-\frac{4}{\pi}\right) x+\left(\frac{4}{k \pi}-1\right)\right) \text { for } x \leq 0.5, \\
\sin (k \pi y)(1-x)\left(\left(2+\frac{4}{k \pi}\right) x-1\right) \text { for } x \geq 0.5 .
\end{array}\right.
\end{gathered}
$$

In both cases the functions $u, \varphi(z, \nabla u) \cdot \boldsymbol{n}$ are continuous across the interface $\partial \overline{\Omega_{1}} \cap \partial \overline{\Omega_{2}}$. The source terms is then computed by $f=-\operatorname{div}(\varphi(z, \nabla u))$. For large values of $\lambda$, test 1 provides an example of large jump of the gradient. 

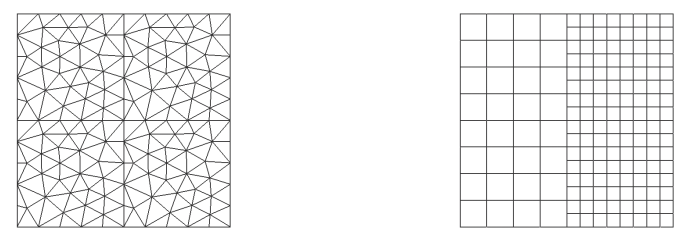

Fig. 3. Example of meshes : mesh 1 (left), mesh 2 (right)

Table 1. Norm of the error for test 1 on mesh 1 with $p_{1}=2, p_{2}=4, \lambda=5.0$

\begin{tabular}{lcccc}
\hline mesh size & $\begin{array}{c}\mathrm{DDFV} \\
L^{\boldsymbol{p}}(\Omega)\end{array}$ & $\begin{array}{c}\mathrm{m}-\mathrm{DDFV} \\
L^{\boldsymbol{p}}(\Omega)\end{array}$ & $\begin{array}{c}\mathrm{DDFV} \\
W^{1, \boldsymbol{p}}(\Omega)\end{array}$ & $\begin{array}{c}\mathrm{m}-\mathrm{DDFV} \\
W^{1, \boldsymbol{p}}(\Omega)\end{array}$ \\
\hline $7.25 \mathrm{E}-02$ & $4.70 \mathrm{E}-01$ & $3.61 \mathrm{E}-02$ & $2.5 \mathrm{E}+01$ & 1.41 \\
$3.63 \mathrm{E}-02$ & $2.36 \mathrm{E}-01$ & $9.14 \mathrm{E}-02$ & $2.03 \mathrm{E}+01$ & $6.62 \mathrm{E}-01$ \\
$1.81 \mathrm{E}-02$ & $1.19 \mathrm{E}-01$ & $2.24 \mathrm{E}-03$ & $1.65 \mathrm{E}+01$ & $3.11 \mathrm{E}-01$ \\
$9.07 \mathrm{E}-03$ & $6.01 \mathrm{E}-02$ & $4.46 \mathrm{E}-04$ & $1.34 \mathrm{E}+01$ & $1.47 \mathrm{E}-01$ \\
\hline
\end{tabular}

Table 2. Norm of the error for test 1 on mesh 2 for $p_{1}=2, p_{2}=4, \lambda=5.0$

\begin{tabular}{lcccc}
\hline mesh size & $\begin{array}{c}\text { DDFV } \\
L^{\boldsymbol{p}}(\Omega)\end{array}$ & $\begin{array}{c}\mathrm{m}-\mathrm{DDFV} \\
L^{\boldsymbol{p}}(\Omega)\end{array}$ & $\begin{array}{c}\mathrm{DDFV} \\
W^{1, \boldsymbol{p}}(\Omega)\end{array}$ & $\begin{array}{c}\mathrm{m}-\mathrm{DDFV} \\
W^{1, \boldsymbol{p}}(\Omega)\end{array}$ \\
\hline $8.83 \mathrm{E}-02$ & $9.62 \mathrm{E}-01$ & $9.93 \mathrm{E}-02$ & $3.26 \mathrm{E}+01$ & $2.52 \mathrm{E}+00$ \\
$4.41 \mathrm{E}-02$ & $4.82 \mathrm{E}-01$ & $2.52 \mathrm{E}-02$ & $2.62 \mathrm{E}+01$ & $1.01 \mathrm{E}+00$ \\
$2.21 \mathrm{E}-02$ & $2.44 \mathrm{E}-01$ & $6.31 \mathrm{E}-03$ & $2.12 \mathrm{E}+01$ & $4.09 \mathrm{E}-01$ \\
$1.10 \mathrm{E}-02$ & $1.23 \mathrm{E}-01$ & $1.58 \mathrm{E}-03$ & $1.71 \mathrm{E}+01$ & $1.64 \mathrm{E}-01$ \\
\hline
\end{tabular}

Tables 1 and 2 show that the DDFV method is first order in $L^{p}$ norm whereas the m-DDFV is second order for both meshes (see Fig. 3). Note that the order of the m-DDFV in $W^{1, \boldsymbol{p}}$ norm is better on the mesh 2 (1.31) which is refined in the subdomain where $p=4$ than for the regular triangular one (mesh $1: 1.07$ ). Table 3 gives the convergence orders of the m-DDFV scheme for the test 2 for various values of $\left(p_{1}, p_{2}\right)$ on the mesh 2 . In this test the solution depends on both variables $x$ and $y$, but $\nabla u$ is continuous at the interface which explains that DDFV and m-DDFV schemes have a similar behavior. Eventhough we get analogous convergence rate for $\left(p_{1}=2, p_{2}=4\right)$ and $\left(p_{1}=4, p_{2}=2\right)$, smaller global error is obtained in the case when the mesh is more refined in the domain where $p_{i}$ is big.

\section{Conclusions}

We propose here a finite volume approach to approximate nonlinear transmission problems on general 2D grids. The m-DDFV scheme we study is solved 
by means of a decomposition-coordination method. Numerical results in the case of $p$ - laplacian like operators illustrate the good behavior of the scheme especially in case of big jumps of the gradient.

Table 3. Convergence rates in the two domains $\Omega_{1}$ and $\Omega_{2}$ for test 2 with $k=5$

\begin{tabular}{lcccc} 
& $L^{p_{1}}\left(\Omega_{1}\right)$ & $L^{p_{2}}\left(\Omega_{2}\right)$ & $W^{1, p_{1}}\left(\Omega_{1}\right)$ & $W^{1, p_{2}}\left(\Omega_{2}\right)$ \\
\hline$p_{1}=2, p_{2}=1.5$ & 2.00 & 1.99 & 1.49 & 1.69 \\
$p_{1}=2, p_{2}=4$ & 2.00 & 2.00 & 1.56 & 1.20 \\
$p_{1}=4, p_{2}=2$ & 2.04 & 1.98 & 1.20 & 1.60 \\
$p_{1}=3, p_{2}=4$ & 2.11 & 2.02 & 1.30 & 1.19 \\
\hline
\end{tabular}

\section{References}

B. Andreianov, F. Boyer, and F. Hubert. Discrete duality finite volume schemes for Leray-Lions type elliptic problems on general 2D-meshes. Num. Meth. for PDEs, 23(1):145-195, 2007.

F. Boyer and F. Hubert. Finite volume method for $2 \mathrm{~d}$ linear and nonlinear elliptic problems with discontinuities. SINUM, 2007. http://hal. archives-ouvertes.fr/hal-00110436.

Y. Coudière, J.-P. Vila, and P. Villedieu. Convergence rate of a finite volume scheme for a two-dimensional convection-diffusion problem. M2AN Math. Model. Numer. Anal., 33(3):493-516, 1999.

K. Domelevo and P. Omnes. A finite volume method for the Laplace equation on almost arbitrary two-dimensional grids. M2AN Math. Model. Numer. Anal., 39(6):1203-1249, 2005.

M. Feistauer and V. Sobotíková. Finite element approximation of nonlinear elliptic problems with discontinuous coefficients. RAIRO Modél. Math. Anal. Numér., 24(4):457-500, 1990.

R. Glowinski. Numerical methods for nonlinear variational problems. Springer Series in Computational Physics. Springer-Verlag, New York, 1984.

F. Hermeline. Approximation of diffusion operators with discontinuous tensor coefficients on distorted meshes. Comput. Methods Appl. Mech. Engrg., 192 (16-18):1939-1959, 2003.

W. B. Liu. Degenerate quasilinear elliptic equations arising from bimaterial problems in elastic-plastic mechanics. Nonlinear Anal., 35(4):517-529, 1999.

W. B. Liu. Finite element approximation of a nonlinear elliptic equation arising from bimaterial problems in elastic-plastic mechanics. Numer. Math., 86(3):491-506, 2000. 